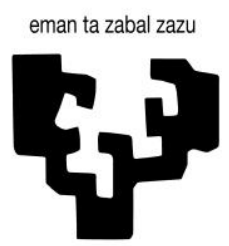

Universidad Euskal Herriko del País Vasco Unibertsitatea

\title{
ADAPTIVE SCALABLE SVD UNIT FOR FAST PROCESSING OF LARGE LSE PROBLEMS
}

IÑAKI BILDOSOLA \& UNAI MARTINEZ-CORRAL KOLDO BASTERRETXEA 


\section{MOTIVATION}

\section{-Motivation}

-Selecting the Algorithm -Selecting SVD Method -Speaking About the Accuracy -Improving Previous Work -Results

- HW FPGA Implementation -Why and How Scalable? -Conclusion -Future Work
- Previous Project

- Computational Intelligence applications

- Real Time Computation

- LSE problems

- Resulting Matrices:

- Large-scale

- Rank-deficient

- Ill-conditioned matrices

- Implementation in MicroBlaze $\rightarrow$ Too Much Delay

- Need for acceleration $\rightarrow$ parallel processing \& optimized faster implementation $\rightarrow$ FPGA 


\section{SELECTING THE ALGORITHM WHY SVD?}

\begin{tabular}{|l|}
\hline -Motivation \\
-Selecting the \\
Algorithm \\
-Selecting SVD \\
Method \\
-Speaking \\
About the \\
Accuracy \\
-Improving \\
Previous Work \\
-Results \\
- HW FPGA \\
Implementation \\
-Why and How \\
Scalable? \\
-Conclusion \\
-Future Work
\end{tabular}

- We needed an algorithm numerically robust

- Struggling with deficient matrices

- Struggling with non-square matrices

- Avoid the Inverse calculation

- Obtain the Pseudoinverse

- Good Base for Problem Reduction (future Work) 


\section{SELECTING SVD METHOD WHY ONE-SIDED JACOBI?}

-Motivation -Selecting the Algorithm -Selecting SVD Method

-Speaking About the Accuracy -Improving Previous Work -Results

- HW FPGA Implementation -Why and How Scalable? -Conclusion -Future Work
- Easily Parallelizable $\rightarrow$ Jacobi

- What more?

- Purely non-conflicting $\rightarrow$ one-sided

- Optimizing the managed unit size $\rightarrow$ onesided

- Main features

- Based on Column Pairs Orthogonalization

- Given's rotations by Rutishauser formulas

$$
\tan \left(2 \Theta_{i j}^{k}\right)=\frac{2 *\left(A_{:, i}^{k} * A_{:, j}^{k}\right)}{\left\|A_{:, j}^{k}\right\|^{2}-\left\|A_{:, i}^{k}\right\|^{2}} \quad A V=W \quad A=U S V^{T}
$$




\section{SPEAKING ABOUT THE ACCURACY IMPOSSED CONDITIONS}

-Motivation -Selecting the Algorithm -Selecting SVD Method

-Speaking About the Accuracy -Improving Previous Work -Results - HW FPGA Implementation -Why and How Scalable? -Conclusion -Future Work
- Computing Precisions $\rightarrow$ Sets The Maximum

- Matrix Conditioning $\rightarrow$ Impacts on the Accuracy

- $\mathrm{K}(\mathrm{A})=10^{k} ; \mathrm{CP}=10^{m} ;$ Solution $=10^{m-k}$.

- Matrix Size $\rightarrow$ Posible Accumulated error.

- $\mathrm{K}(\mathrm{A})$ \& Matrix Size Impact close to $\mathrm{CP} \rightarrow \mathrm{No}$ Solution or very degraded 


\section{SPEAKING ABOUT THE ACCURACY: IMPOSSED CONDITIONS}

-Motivation -Selecting the Algorithm -Selecting SVD Method

-Speaking About the Accuracy -Improving Previous Work -Results

- HW FPGA Implementation -Why and How Scalable? -Conclusion -Future Work
- Randomly generated matrices $f($ Size, $k(A))$

- Errors: Our Algorithm (') in Single Vs Matlab(") in double

- Singulars $=$ Maximum Normalized Error $=\frac{\sigma^{\prime}-\sigma^{\prime \prime}}{\sigma^{\prime \prime}}$

- Inverse $=\left\|A_{i n v}^{\prime}-A_{i n v}^{\prime \prime}\right\|^{2}$

- Remainder $=\|A-S V D\|^{2}$

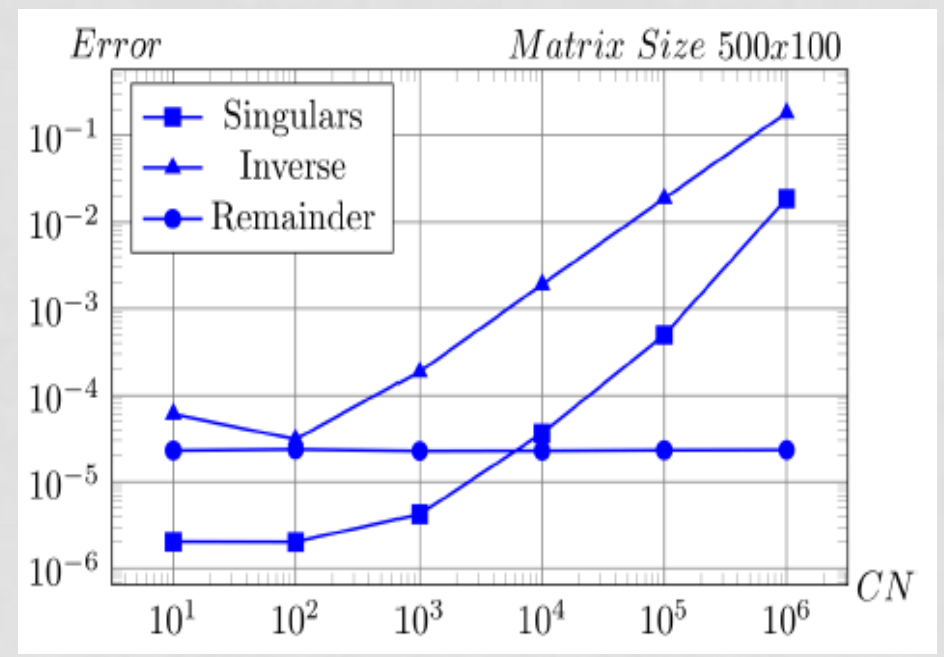




\section{SPEAKING ABOUT THE ACCURACY DECIDED CONDITIONS}

-Motivation -Selecting the Algorithm -Selecting SVD Method

-Speaking About the Accuracy -Improving Previous Work -Results

- HW FPGA Implementation -Why and How Scalable? -Conclusion -Future Work

\section{- Threshold Value}

- Iterative Algorithm Finisher $\rightarrow$ Orthogonalization

- User Defined Parameter $\rightarrow$ Time \& Accuracy Trade-off

- Error Saturation Phenomenon $\rightarrow$ (Imposed Conditions)

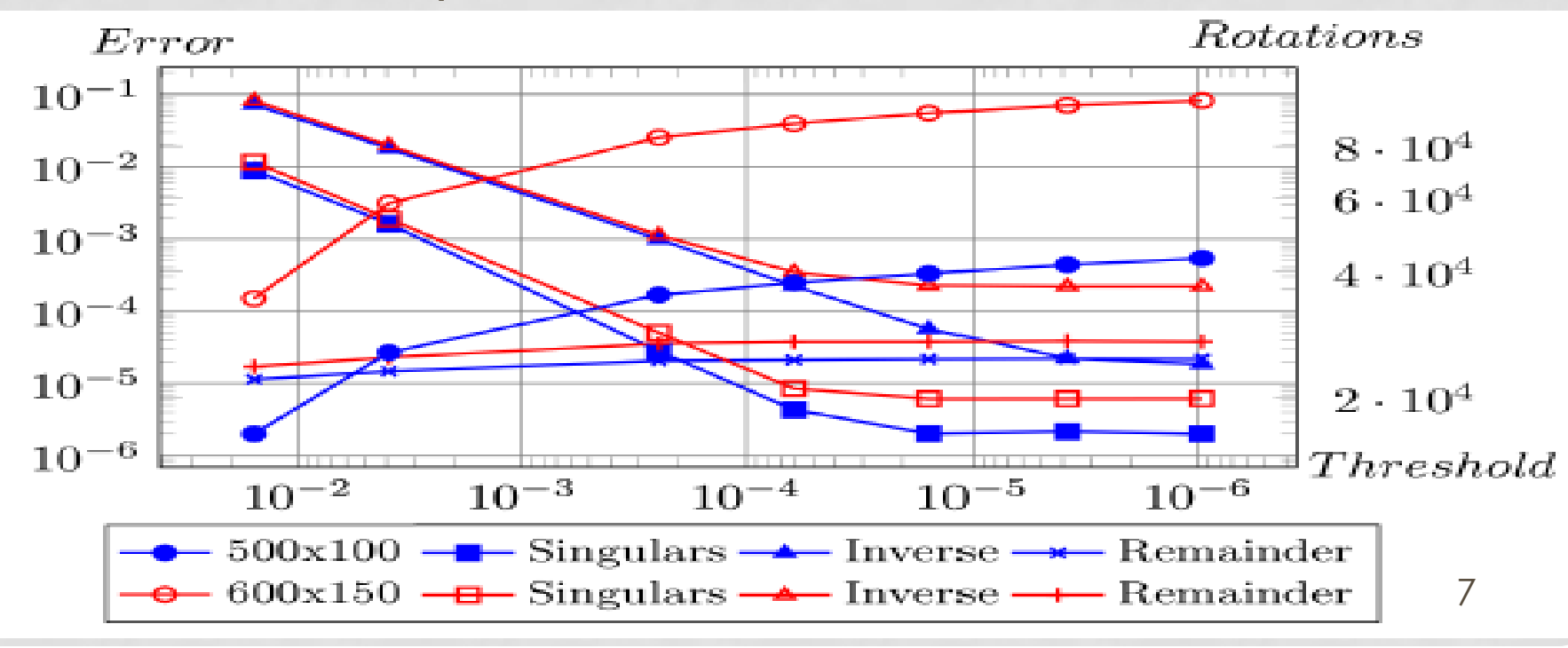




\section{IMPROVING PREVIOUS WORK LEARNING FROM OTHERS}

-Motivation -Selecting the Algorithm -Selecting SVD Method -Speaking About the Accuracy -Improving Previous Work -Results

- HW FPGA Implementation -Why and How Scalable? -Conclusion -Future Work
- Brent and Luck

- Highlighted the column norm importance

- Normalized the Threshold $\rightarrow$ Adapting to the columns' norm $\rightarrow$ Actually calculating the cosine: $\frac{A_{i} \cdot A_{j}^{T}}{\left\|A_{i}\right\|\left\|A_{j}\right\|}<$ Threshold

- Hestenes

- Swap the columns $\rightarrow$ Active Sorting $\rightarrow$ f(column norm) 


\section{IMPROVING PREVIOUS WORK ADDING OUR TOUCH}

-Motivation -Selecting the Algorithm -Selecting SVD Method -Speaking About the Accuracy -Improving Previous Work -Results

- HW FPGA Implementation -Why and How Scalable? -Conclusion -Future Work
- Increased Adaptability

- Realizing that the "Inverse Error" lies on small columns

- Being Fussier with them $\rightarrow$ Harder Threshold

- With Easier Threshold $\rightarrow$ Same Solution Accuracy

- Not rotating in vain the big columns

- AMN:

- $\frac{A_{i} \cdot A_{j}^{T}}{\left\|A_{i}\right\|\left\|A_{j}\right\|}=\cos \left(A_{i}, A_{j}\right)<$ Threshold $\cdot \min \left(\left\|A_{i}\right\|,\left\|A_{j}\right\|\right)$

- AAMN:

- $\frac{A_{i} \cdot A_{j}^{T}}{\left\|A_{i}\right\|\left\|A_{j}\right\|}=\cos \left(A_{i}, A_{j}\right)<$ Threshold $\cdot\left\|A_{j}\right\|$ 


\section{IMPROVING PREVIOUS WORK BEING HW FRIENDLY}

-Motivation -Selecting the Algorithm -Selecting SVD Method -Speaking About the Accuracy -Improving Previous Work -Results

- HW FPGA Implementation -Why and How Scalable? -Conclusion -Future Work
- Initially Two Angles Calculation:

- The Decision $\rightarrow \mathrm{f}$ (cosine)

- The Rotation $\rightarrow$ f(Rutishauser)

- Cos \& RutisHauser $\rightarrow$ both f/columns and its norms)

- Killing two bird with one stone Decision and rotation $\rightarrow f($ RutisHauser)

- Readaptation $\rightarrow$ More sensitive

- AARH: $\theta_{i j}<$ Threshold $\cdot\left\|A_{j}\right\|^{2}$

- Avoiding root squares 


\section{RESULTS \\ THE MODIFIED ONE-SIDED JACOBI}

-Motivation

-Selecting the Algorithm -Selecting SVD Method

-Speaking

About the

Accuracy -Improving Previous Work

-Results

- HW FPGA Implementation -Why and How Scalable? -Conclusion -Future Work

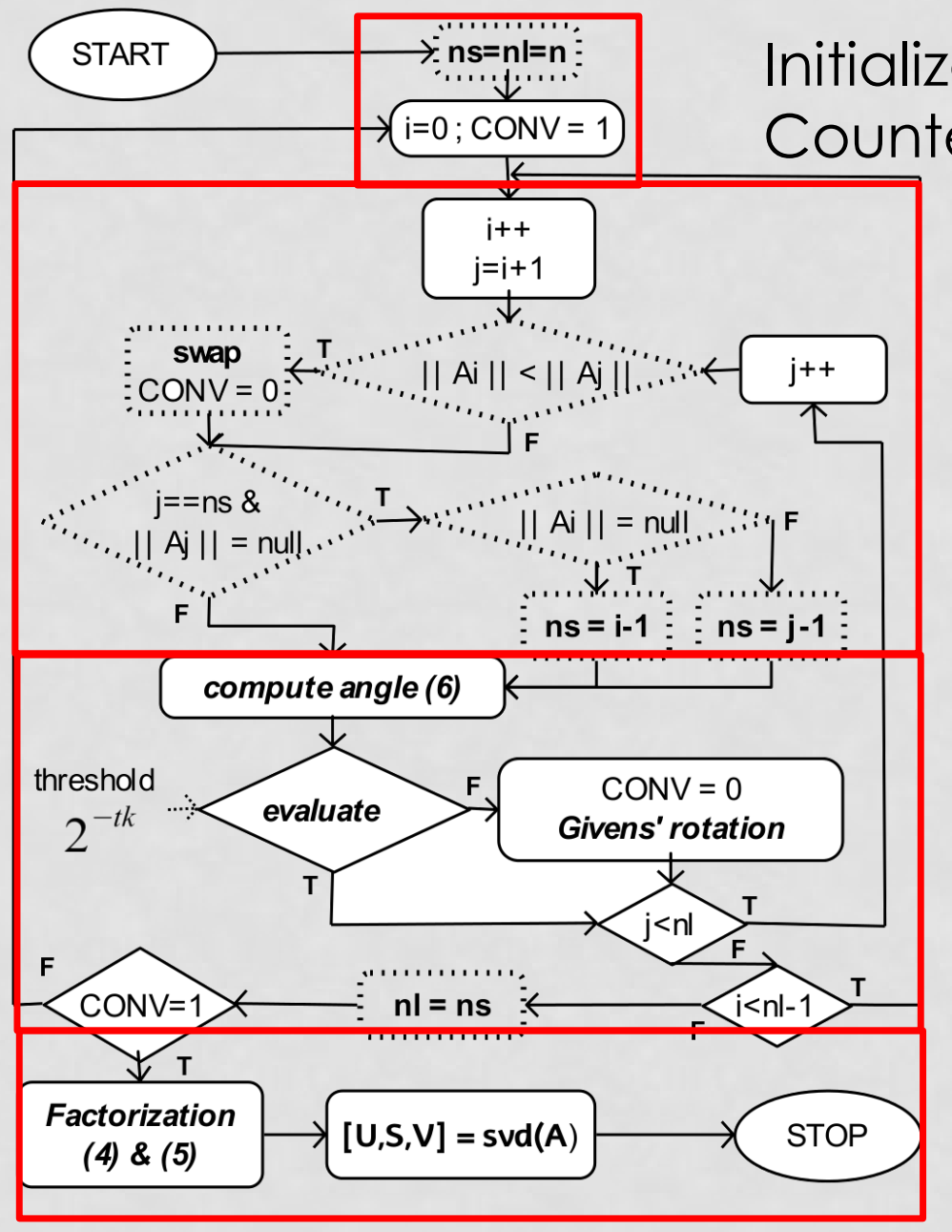

Iteration : Swap \&Null Columns Management

Iteration : Decision, Rotation \& Actualization

Finish: Matrix Factorization $\sigma_{i}=\left\|W_{(:, i)}\right\| \quad U_{(:, i)}=\frac{W_{(:, i)}}{\sigma_{i}}$ 


\section{COMPARATION ANALYSIS TOOLBOX}

Column Arrangement Selection of the Comparation
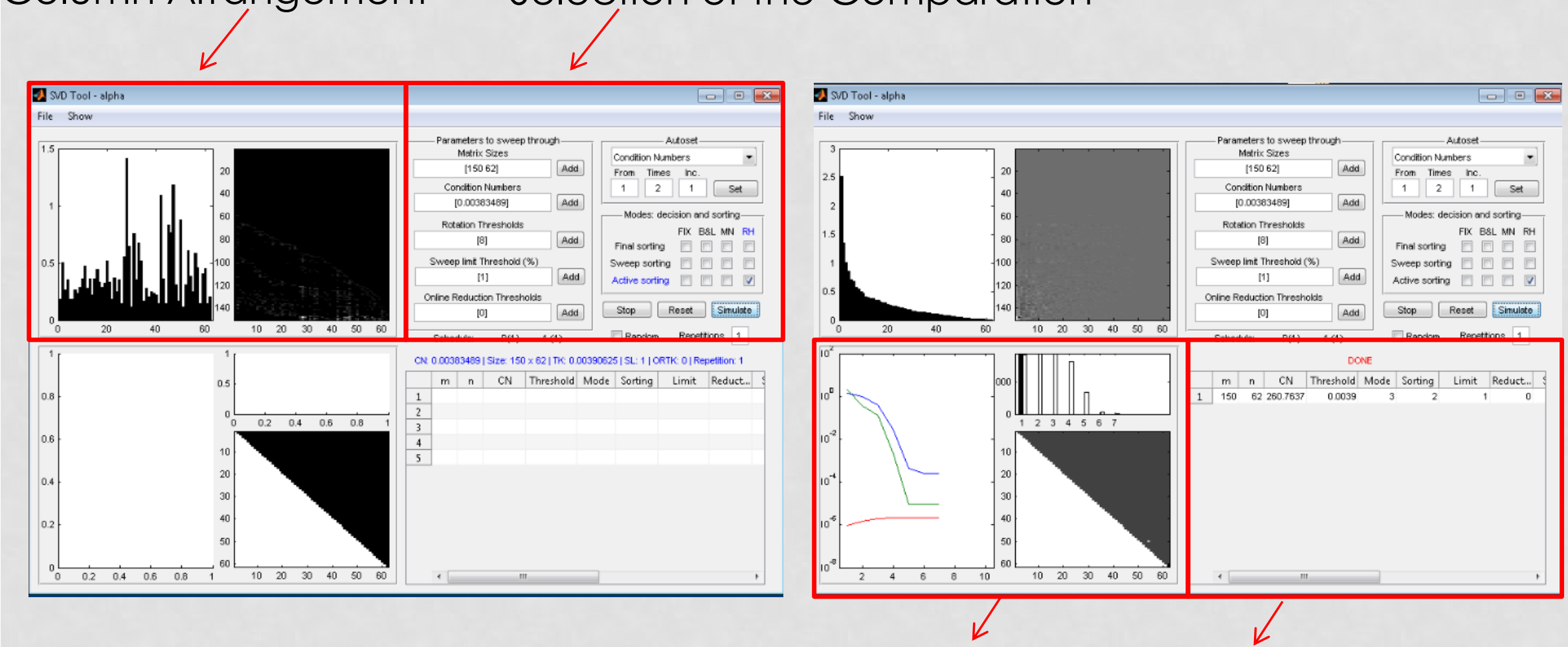

Error Evolution

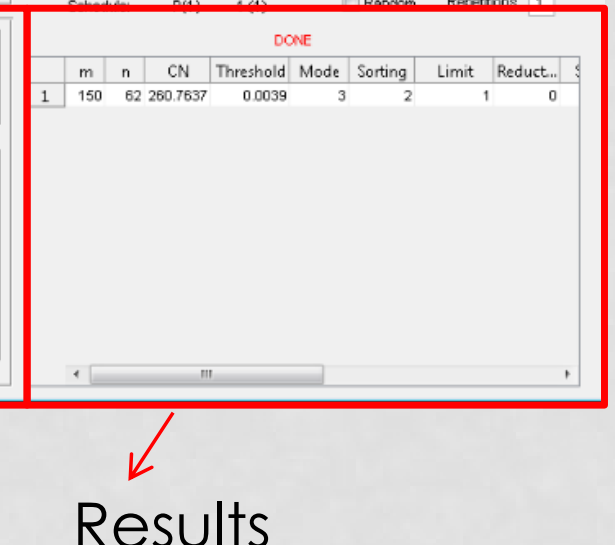




\section{RESULTS \\ COMPARING WITH THE REST}

Obtaining Same Accuracy $\longrightarrow$ Easier Threshold

-Motivation -Selecting the Algorithm -Selecting SVD Method -Speaking About the Accuracy -Improving Previous Work

\section{-Results}

- HW FPGA Implementation -Why and How Scalable? -Conclusion -Future Work
TABLE I: Comparative of algorithm performance

Inverse Error | Threshold

\begin{tabular}{|c|c|c|c|c|}
\hline \\
\hline Fixed & $4.19 \mathrm{E}-6 \mid 24$ & $2.11 \mathrm{E}-5 \mid 24$ & $2.54 \mathrm{E}-4 \mid 24$ & $1.89 \mathrm{E}-3 \mid 24$ \\
\hline B\&L & $3.98 \mathrm{E}-6 \mid 22$ & $1.93 \mathrm{E}-5 \mid 18$ & $1.62 \mathrm{E}-4 \mid 16$ & $1.87 \mathrm{E}-5 \mid 16$ \\
\hline$A B L$ & $3.68 \mathrm{E}-6 \mid 22$ & $1.83 \mathrm{E}-5 \mid 18$ & $1.63 \mathrm{E}-4 \mid 16$ & $1.78 \mathrm{E}-3 \mid 12$ \\
\hline AAMN & $5.60 \mathrm{E}-6 \mid 20$ & $1.76 \mathrm{E}-5 \mid 16$ & $1.68 \mathrm{E}-4 \mid 10$ & $1.34 \mathrm{E}-3 \mid 8$ \\
\hline AARH & $6.77 \mathrm{E}-6 \mid 20$ & $1.87 \mathrm{E}-5 \mid 16$ & $1.76 \mathrm{E}-4 \mid 10$ & $1.41 \mathrm{E}-3 \mid 8$ \\
\hline$\kappa(\mathrm{A})$ & $1,00 \mathrm{E}+01$ & $1,00 \mathrm{E}+02$ & $1,00 \mathrm{E}+03$ & $1,00 \mathrm{E}+04$ \\
\hline Fixed & $33,696 \mid 11.3$ & $33,530 \mid 10.50$ & $33,905 \mid 10.50$ & $34,004 \mid 10.75$ \\
\hline B\&L & $32,303 \mid 9.70$ & $30,911 \mid 10.20$ & $29,858 \mid 10.15$ & $26,482 \mid 10.13$ \\
\hline$A B L$ & $26,096 \mid 8.20$ & $24,356 \mid 8.00$ & $23,121 \mid 8.05$ & $19,927 \mid 7.73$ \\
\hline AAMN & $25,275 \mid 8.13$ & $23,209 \mid 8.05$ & $18,353 \mid 8.05$ & $15,327 \mid 8.30$ \\
\hline AARH & $25,512 \mid 8.40$ & $23,345 \mid 8.50$ & $18,960 \mid 8.53$ & $16,078 \mid 8.35$ \\
\hline
\end{tabular}

TABLE II

TEST MATRICES RESULTS

Inverse Error $\mid 2^{- \text {Threshold }}$; Rotations $\mid$ Sweeps

\begin{tabular}{|c|c|c|c|}
\hline $\begin{array}{c}\text { Size } \mid \kappa \\
\text { Name }\end{array}$ & $\begin{array}{c}201 \times 47 \mid 7.5 \mathrm{E} 1 \\
\text { JGD_Kocay/Trec9 }\end{array}$ & $\begin{array}{c}235 \times 216 \mid 1.7 \mathrm{E} 3 \\
\text { JGD_Forest/TF11 }\end{array}$ & $\begin{array}{c}200 \times 200 \mid 2.4 \mathrm{E} 3 \\
\text { Bai/bwm200 }\end{array}$ \\
\hline \multirow{2}{*}{ ABL } & $1.99 \mathrm{E} 6 \mid 22$ & $1.4 \mathrm{E}-3 \mid 12$ & $2.96 \mathrm{E} 4 \mid 14$ \\
& $5,004 \mid 7$ & $97,281 \mid 9$ & $95,902 \mid 9$ \\
\hline \multirow{2}{*}{ AAMN } & $1.96 \mathrm{E}-6 \mid 18$ & $5.3 \mathrm{E} 4 \mid 6$ & $1.43 \mathrm{E} 4 \mid 8$ \\
& $4,903 \mid 8$ & $75,433 \mid 9$ & $61,981 \mid 9$ \\
\hline \multirow{2}{*}{ AARH } & $2.21 \mathrm{E} 6 \mid 16$ & $8.03 \mathrm{E}-4 \mid 4$ & $5.64 \mathrm{E}-4 \mid 6$ \\
& $4,908 \mid 9$ & $76,854 \mid 9$ & $57,675 \mid 10$ \\
\hline
\end{tabular}

Obtaining Same Accuracy $\longrightarrow$ Less Rotations

Obtaining a Better Result $\rightarrow$ The Higher the K(A)

Testing With Real Matrices \& Obtaining Expected Results 


\section{RESULTS \\ COMPARING WITH THE REST}

\begin{tabular}{|l|}
\hline -Motivation \\
-Selecting the \\
Algorithm \\
-Selecting SVD \\
Method \\
-Speaking \\
About the \\
Accuracy \\
-Improving \\
Previous Work \\
-Results \\
- HW FPGA \\
Implementation \\
-Why and How \\
Scalable? \\
-Conclusion \\
-Future Work \\
\end{tabular}

- Savings in Number of Rotations

\begin{tabular}{|c|c|c|c|}
\hline CN & Fixed to AARH & B\&L to AARH & ABL to AARH \\
\hline $1,00 \mathrm{E}+01$ & $24,29 \%$ & $21,02 \%$ & $2,24 \%$ \\
\hline $1,00 \mathrm{E}+02$ & $30,38 \%$ & $24,48 \%$ & $4,15 \%$ \\
\hline $1,00 \mathrm{E}+03$ & $44,08 \%$ & $36,50 \%$ & $18,00 \%$ \\
\hline $1,00 \mathrm{E}+04$ & $52,72 \%$ & $39,29 \%$ & $19,32 \%$ \\
\hline
\end{tabular}




\section{ARCHITECTURE}

\section{-Motivation}

-Selecting the

Algorithm

-Selecting SVD Method

-Speaking

About the

Accuracy

-Improving

Previous Work

-Results

- HW FPGA Implementation

-Why and How

Scalable?

-Conclusion

-Future Work

\section{Double Data-Flow:}

- Primary: Linear array to manage $\mathrm{Ai} / \mathrm{Aj}$

- Secondary: Asynchronous fullduplex shared bus to manage Vi/Vj

- FIFO between PUs
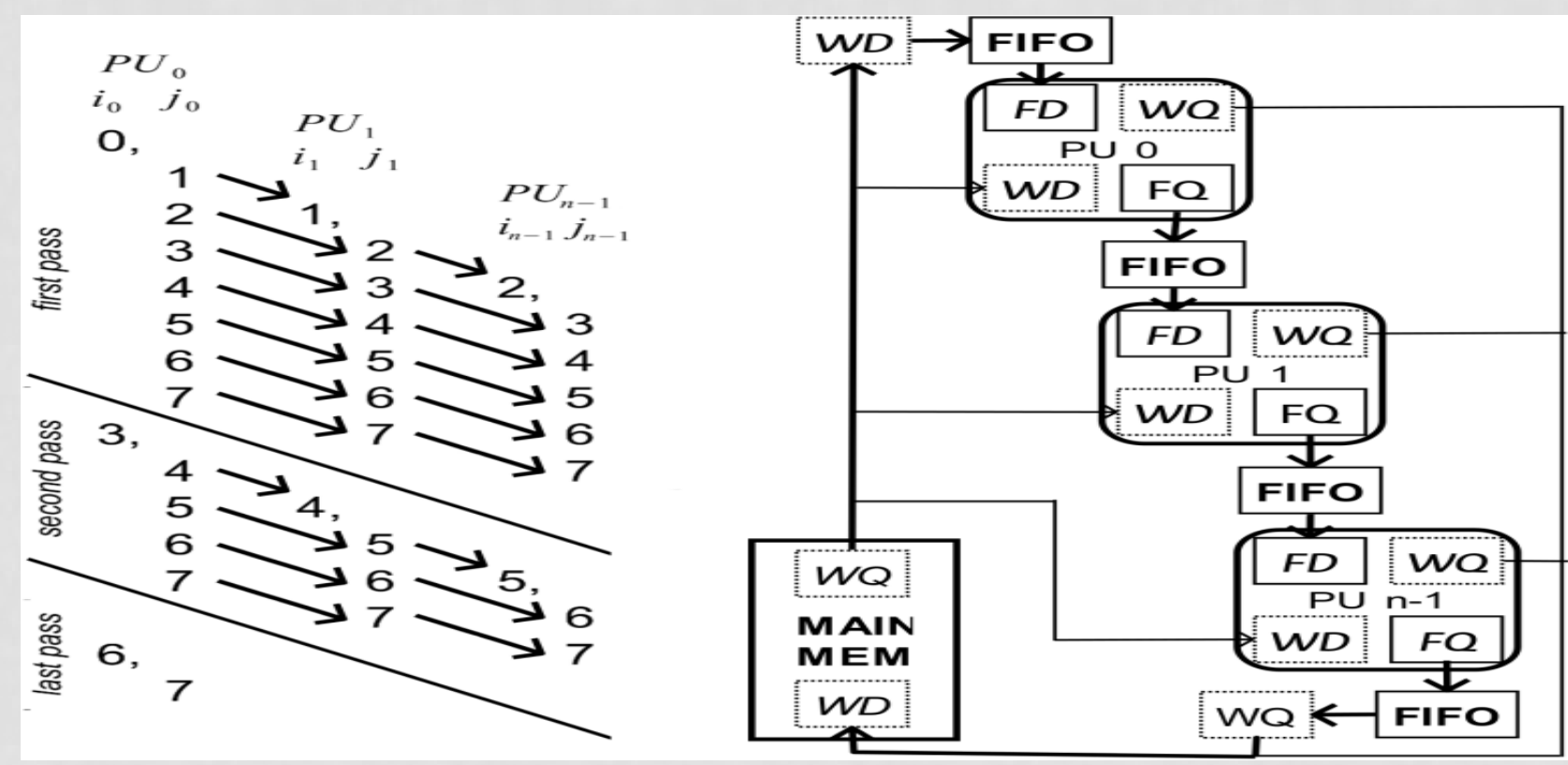


\section{HW FPGA IMPLEMENTATION PROCESSING UNIT}

-Motivation -Selecting the Algorithm -Selecting SVD Method -Speaking About the Accuracy -Improving Previous Work -Results

\section{- HW FPGA} Implementation -Why and How Scalable? -Conclusion -Future Work

\section{PU Design:}

- Evaluation: Computing square Euclidean norms and vector multiplication, swaping and deciding

- Cordic: Theta calculation and rotations' performing

- Cache: $L(m+n)$ and $R(\max (m, 2 n)$

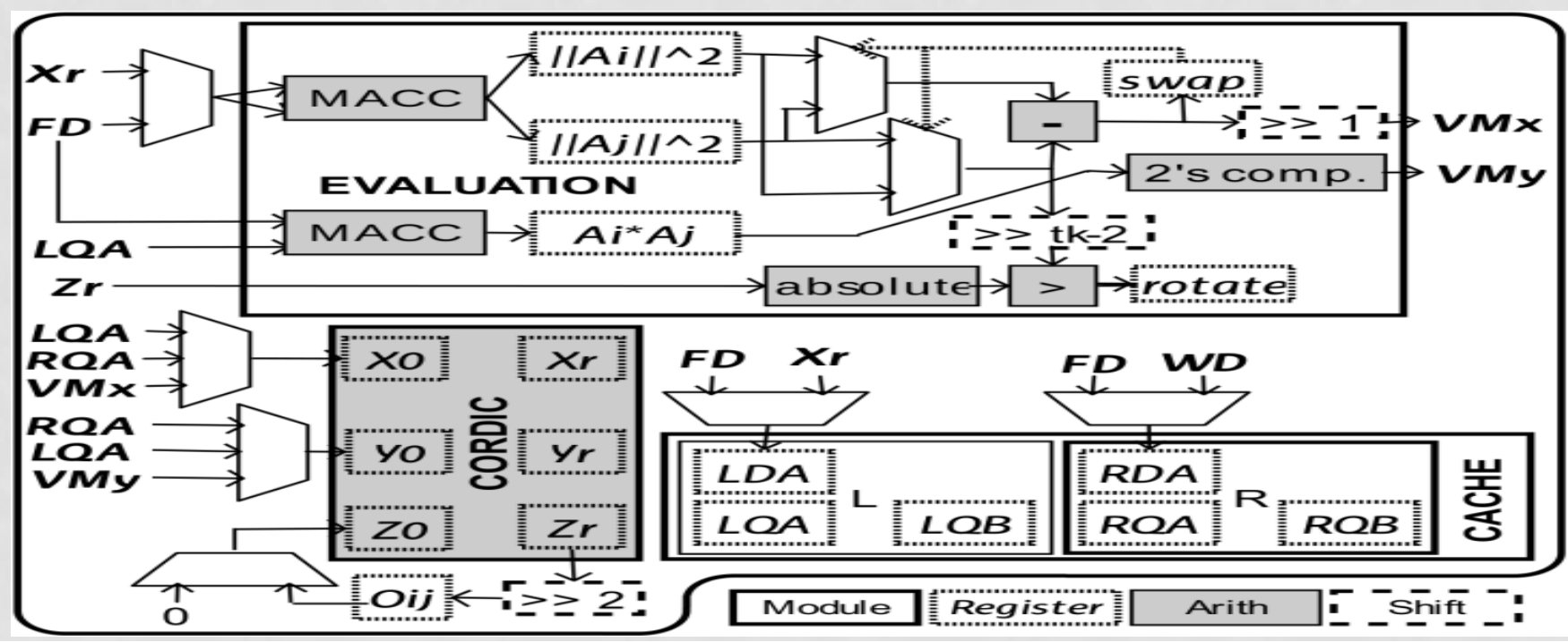




\section{WHY AND HOW SCALABLE?}

-Motivation -Selecting the Algorithm -Selecting SVD Method -Speaking About the Accuracy -Improving Previous Work -Results

- HW FPGA Implementation -Why and How Scalable? -Conclusion -Future Work
- No limited to specific matrices and HW: Generic Solution

- Different sizes

- Different Shapes

- Different budgets

- Architecture

- Based on basics processing units PUs

- PUs variable on quantity

- From 2 to $\mathrm{n} / 2$

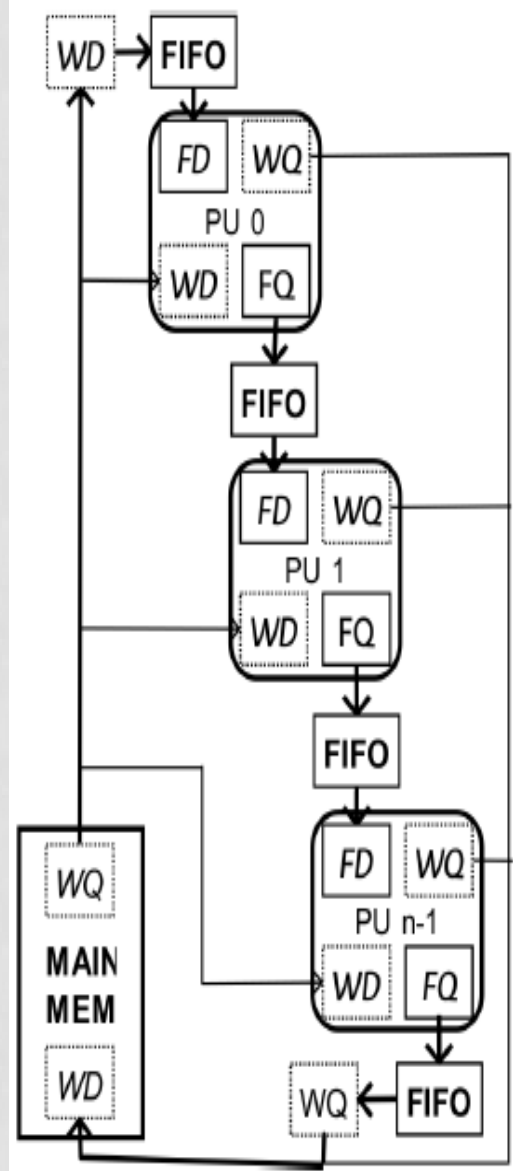




\section{IMPLEMENTATION RESULTS}

\begin{tabular}{|c|c|c|}
\hline & xc6slx45-3figg 484 & xc7k160t-3flog 484 \\
\hline Area & $56 \%$ & $86 \%$ \\
\hline DSPs & $93 \%$ & $60 \%$ \\
\hline RAM & $62 \%$ & $44 \%$ \\
\hline Matrix Size ; K(A) & $300 \times 100 ; 10^{2}$ & $750 \times 250$ \\
\hline PUs & 9 & 60 \\
\hline Frequency & $55 \mathrm{MHz}$ & $90 \mathrm{MHz}$ \\
\hline Processing Time & $60 \mathrm{~ms}$ & \\
\hline
\end{tabular}

Word-Length :18 bits 


\section{CONCLUSION}

- AAMN and AARH proposed outperforming previous proposals.

- Small Columns Important Columns

- Same Accuracy Less Rotations

- User-defined Accuracy -> Threshold

- HW Friendly

- An implemented parallell processing scheme proposed:

- Linear Array of PUs

- Scalable

- Double Data-Flow 


\section{FUTURE WORK}

- Online reduction of problem size

- Improve sorting

- Optimize PU design

- Improved CORDIC realization (Redundant arithmetic (Ercegovac) or Square root and division free (Gotze))

- Ad-hoc online estimators

- Atan

- Square norm 


\section{THANK YOU VERY MUCH}

- Questions or Details? 